\title{
Utilisation des techniques électrochimiques pour la mesure du frottement pariétal dans les écoulements diphasiques
}

\author{
Electrochemical methods for measurement \\ of wall shear stress in two-phase flow
}

Gérard Cognet, Michel Lebouché et Mohamed Souhar*

Position du problème

L'étude des écoulements diphasiques nécessite pour le bilan de quantité de mouvement l'évaluation précise des termes:

1. de pesanteur ; 2 . d'accélération ; 3 . de frottement pariétal ; 4. d'interface.

La mesure directe du frottement pariétal est importante particulièrement dans les régimes suivants :

- écoulement à bulles pour permettre la détermination des termes d'interface,

- écoulement à poches pour mieux interpréter au passage de chaque poche la succession d'un écoulement à film, souvent à contre courant et d'un écoulement à bulles de sillage,

- écoulement annulaire dispersé pour évaluer les échanges gouttelettes-film.

On peut espérer que ces mesures introduites dans les équations générales permettront l'élaboration d'un modèle plus complet des pertes de charge dans les écouments diphasiques.

Principe de la méthode de mesure

La technique utilisée, la vélocimétrie polarographique, est l'équivalent en transfert de masse de l'anémométrie à fil (ou à film) chaud à température constante [1-2]. On utilise l'électrolyse rapide d'un réactif sur une microélectrode affleurant une paroi inerte baignée par un écoulement. Sous certaines conditions (excès d'électrolyte indifférent, potentiel d'électrode correspondant

(*) Laboratoire d'Energétique et de Mécanique Théorique et Appliquée. Institut National Poly technique de Lorraine, 2, rue de la Citadelle 54000 NANCY. au palier de diffusion), le courant limite d'électrolyse I, proportionnel au flux de réactif à la microélectrode est lié à la valeur locale du gradient pariétal de vitesse $S$ et donc de la contrainte à la paroi $\tau=\mu S$ pour un fluide newtonien, selon une loi $I \sim S^{1 / 3}$.
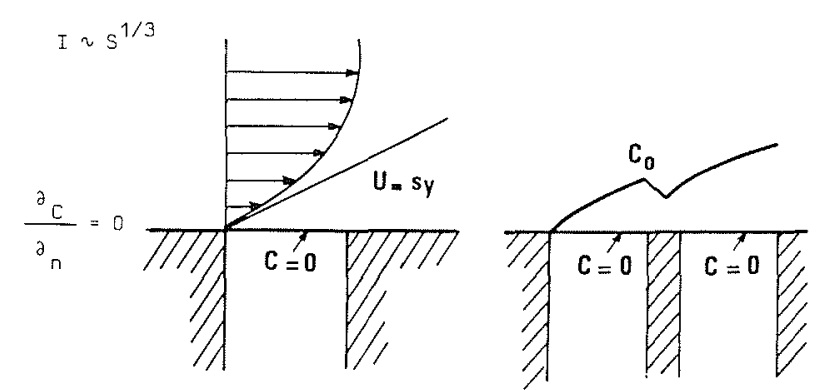

Figure 1.a - Electrodes simple ou double

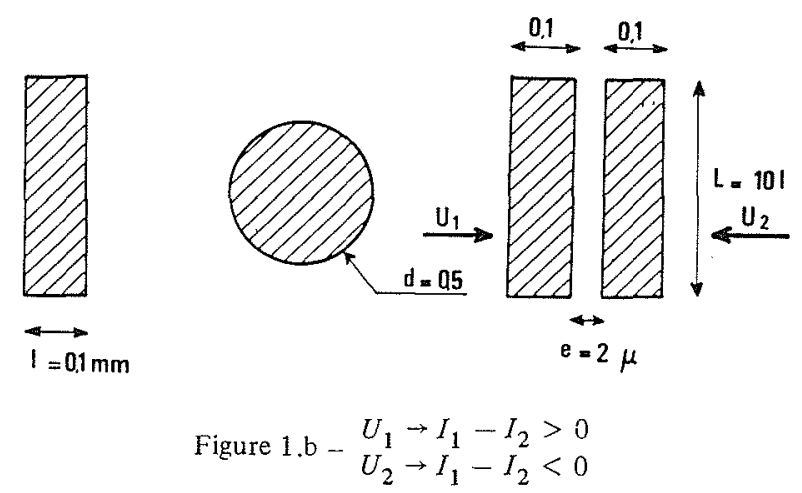

La microsonde est également sensible aux fluctuations de $S$ au cours du temps. En outre l'utilisation de sondes doubles permet de connaitre le sens instantané de l'écoulement du fait du sillage massique d'une électrode sur l'autre. (Fig. 1a et b). 


\section{Dispositif d'essai}

Le montage expérimental comprend : (Fig. 2). - un tube en plexiglas de diamètre intérieur $10 \mathrm{~mm}$ et de longueur $2400 \mathrm{~mm}$

- une pompe dont le corps est en PVC avec un débit maximal de $100 \mathrm{~cm}^{3} / \mathrm{s}$ mesuré par un débimètre à diaphragme

- un banc de distribution de gaz (azote) avec bouteille munie d'un manodétendeur, mesure de débit par rotamètre, et injecteur central

- un élément de tube test portant sur sa paroi interne des sondes polarographiques simples et doubles

- la chaine électronique de mesure adaptée : alimentation des sondes, transformation des courants en tension, système d'enregistrement ou d'acquisition du signal, système de traitement.

\section{Premiers résultats}

\section{Qualitatifs}

Des essais préliminaires ont été effectués pour étudier qualitativement l'enregistrement du passage d'une bulle ou d'un bouchon par les électrodes simple ou double.

Les figures $3 \mathrm{a}$ et $3 \mathrm{~b}$ reproduisent les valeurs obtenues sur un oscilloscope dans le cas d'un débit liquide faible ou nul.
Il apparait que de telles sondes constituent un moyen d'investigation très sensible pour étudier les écoulements diphasiques au voisinage des parois; en particulier les sondes doubles qui permettent de détecter le changement de sens de l'écoulement comme on le constate au passage d'un bouchon et, même, de mettre en évidence les rides sur le film de retour.

\section{Quantitatifs}

Ces résultats sont relatifs à une sonde simple donnant le module du gradient pariétal de vitesse et non son signe lié au sens de l'écoulement (il peut exister des frottements négatifs en régime à poches)

On a fait varier le débit de gaz $Q_{G}$ en gardant le débit liquide $Q_{L}$ constant

- en écoulement liquide seul, pour le débit $Q_{L}$, on mesure le courant $I_{0}$ et la valeur quadratique moyenne de ses fluctuations $\sqrt{i_{0}^{2}}$.

- en écoulement diphasique, on mesure $I$ et $\sqrt{i^{2}}$.

Le module du frottement pariétal $|\tau|$ étant sensiblement proportionnel à $I^{3}$, on peut écrire en première approximation

$$
\frac{|\bar{\tau}|}{\overline{\tau_{0}}} \simeq\left(\frac{\bar{I}}{\bar{I}_{0}}\right)^{3} \quad \text { et } \quad \frac{\sqrt{\bar{\tau}^{2}} \cdot}{\bar{\tau}} \simeq 3 \frac{\sqrt{\bar{i}^{2}}}{\bar{I}}
$$

$\bar{\tau}_{0}$ frottement moyen pour un débit $Q_{L}$ de liquide seul $|\bar{\tau}|$ moyenne du module du frottement pour un débit de liquide $Q_{L}$ et un débit de gaz $Q_{G}$

$\tau^{\prime}$ fluctuation du frottement.

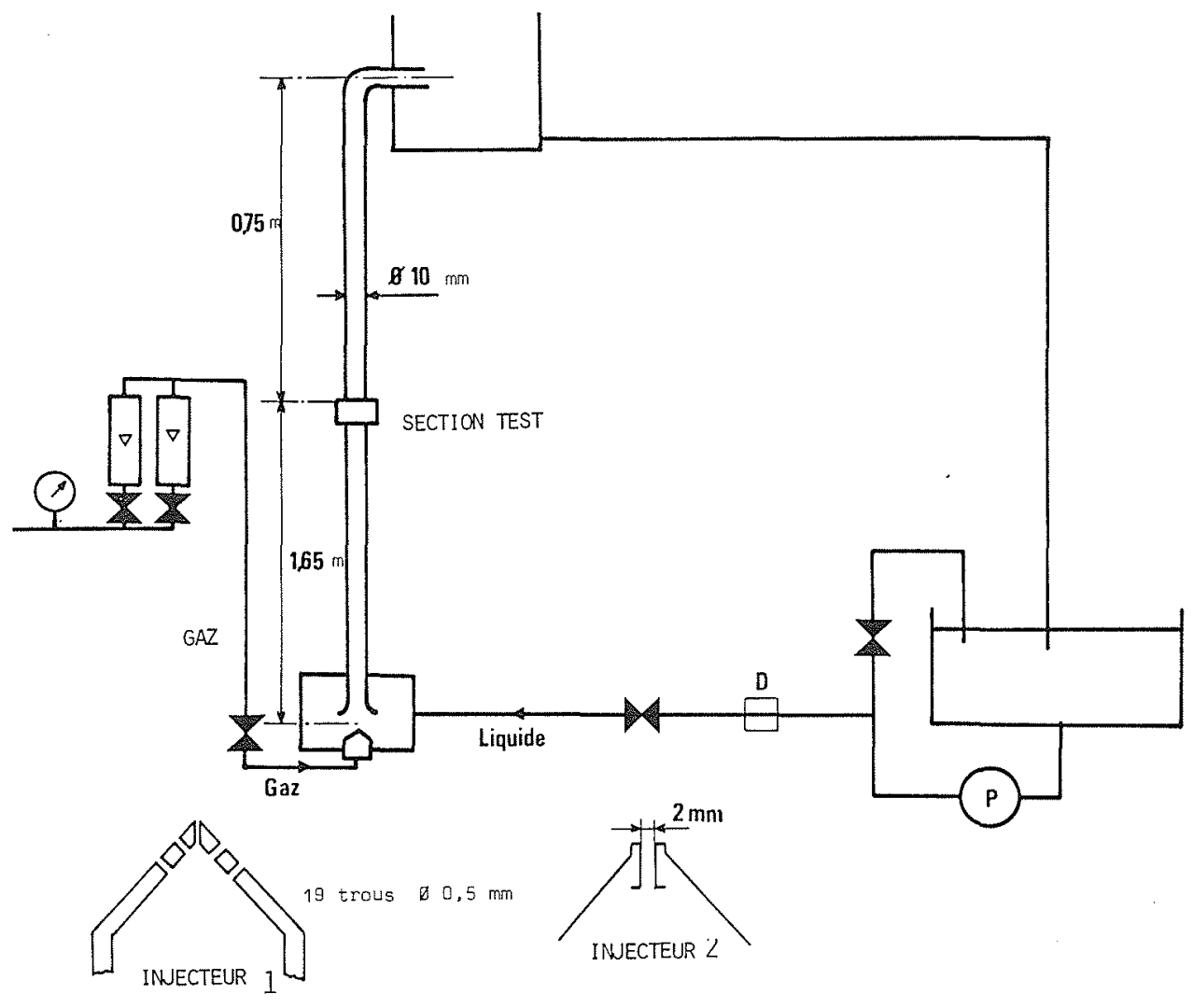

Figure 2 - Dispositif expérimental 
SONDE SIMPLE
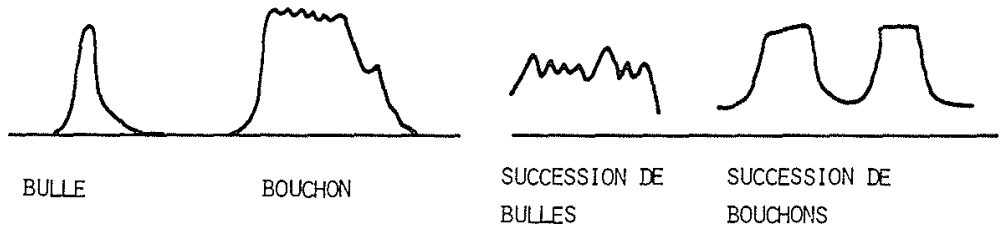

SONDE DOUBLE

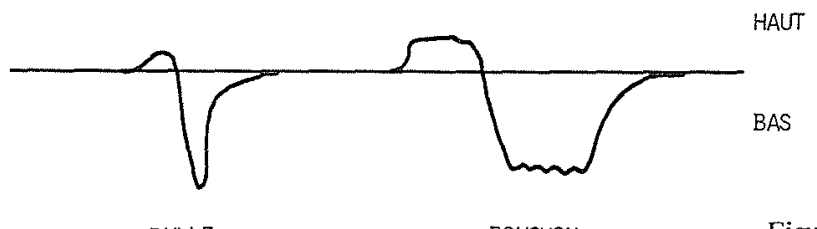

BULE

BOUCHON

Figure 3.a - Débit liquide nul

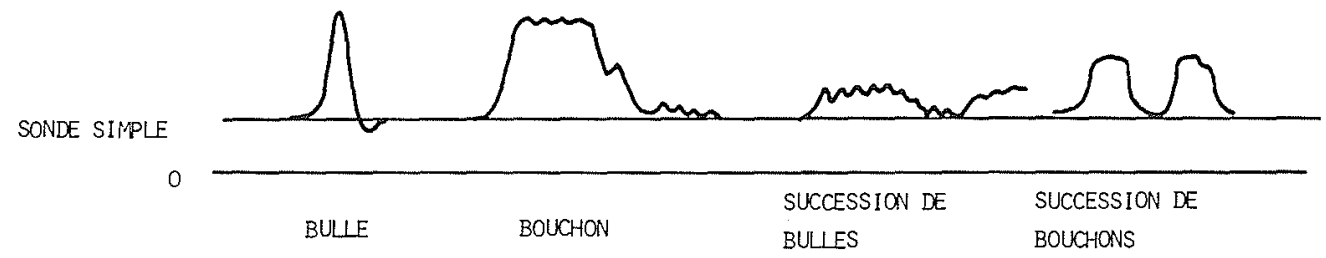

SONDE DOLBLE

0

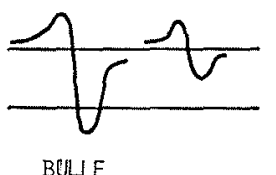

BULE

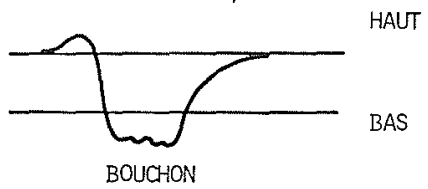

BOUCHON

Figure 3.b - Faible débit liquide

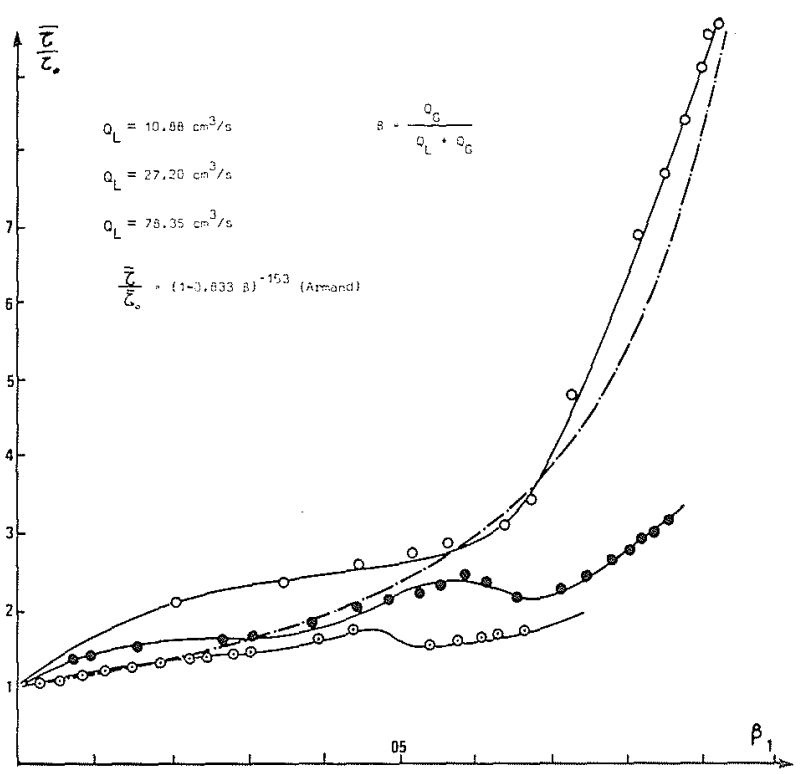

Figure 4

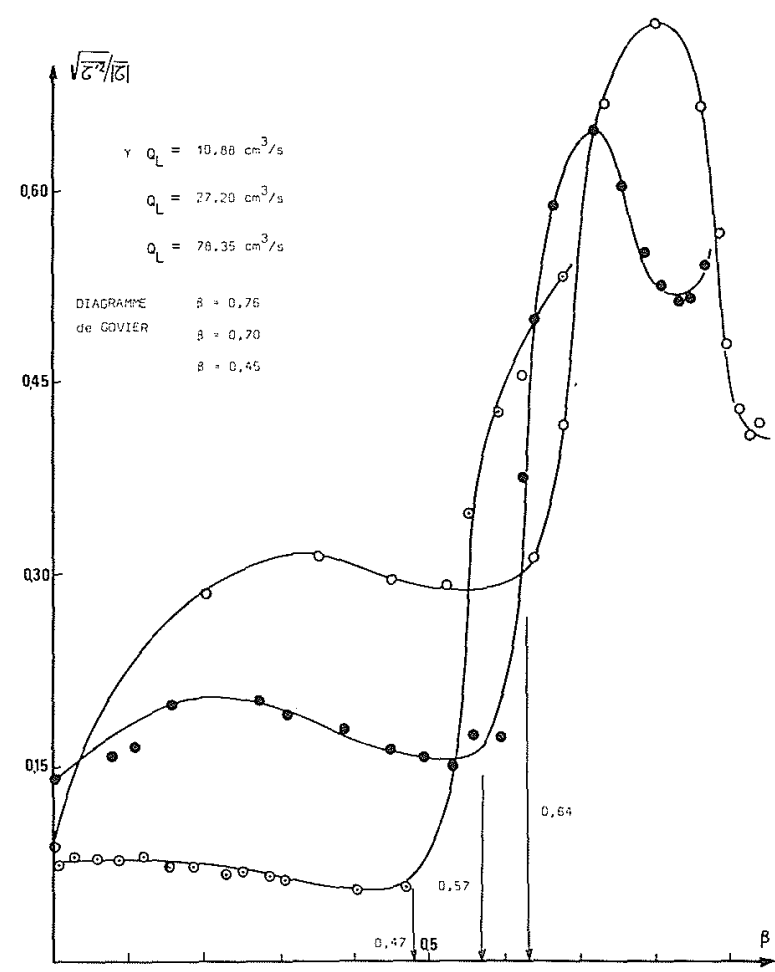

Figure 5 
En définissant $\beta=\frac{Q_{G}}{Q_{L}+Q_{G_{F}}}$, on a tracé respectivement $|\tau| / \tau_{0}=f_{1}(\beta)$ et $\frac{\sqrt{\bar{\tau}^{2}}}{|\bar{\tau}|}=f_{2}(\beta)$ pour les débits liquides $Q_{L}=10,88 ; 27,20$ et $78,35 \mathrm{~cm}^{3}$.

Jusqu'à $\beta=0,6$, les courbes de frottement de la figure 4 semblent assez bien représentées par la corrélation proposée par Armand.

$\bar{\tau} / \bar{\tau}_{0}=(1-0,833 \beta)^{-n}$ avec $n=1,53$. Ceci est confirmé par les mesures de Kutateladze [3] qui trouve $1,53<n<1,75$.

Les fluctuations de frottement sont représentées sur la figure 5. L'accroissement brutal constaté sur chaque courbe indique vraisemblablement le passage du régime à bulles au régime à poches. C'est ainsi que la transition correspondant aux débits précédents est observée respectivement pour $\beta=0,65 ; 0,57$ et 0,47 , alors que le diagramme de Govier [4] donne dans les mêmes conditions $\beta=0,75 ; 0,70$ et 0,45 .

\section{Conclusion}

Ces résultats encourageants sont relatifs à une pre. mière série de mesures ; ils vont être étendus à une large gamme de débits sur la même installation et sur une plus grande $(\varnothing 44 \mathrm{~mm}$ et longueur $7300 \mathrm{~mm})$ construite récemment au LEMTA et réplique d'une installation déjà réalisée au LEMF d'Orsay, l'ensemble des expériences étant menées en concertation entre les deux laboratoires, dans le cadre d'une action thématique programmée du CNRS (mécanique et thermodynamique)

\section{Références}

[1] LEBOUCHE, COGNET - Chimie Industrie, Génie Chimique, Vol. $97, n^{\circ} 12,1967$.

[2] REISS, HANRATTY - A.I.Ch.E, tome 9, p. 154, (1963).

[3| KUTATLLADZI: - Recherches expérimentales des écoulements turbulents (en russe).Moscou. 1975 liditions Nayka.

[4] GOVIER - Canad. J. Chim. Eng. 35, (2) p. 58-70 (1957). 